\title{
Ocean Shipping Container Availability Report
}

\author{
A weekly publication of the Transportation Services Division \\ Agricultural Marketing Service
}

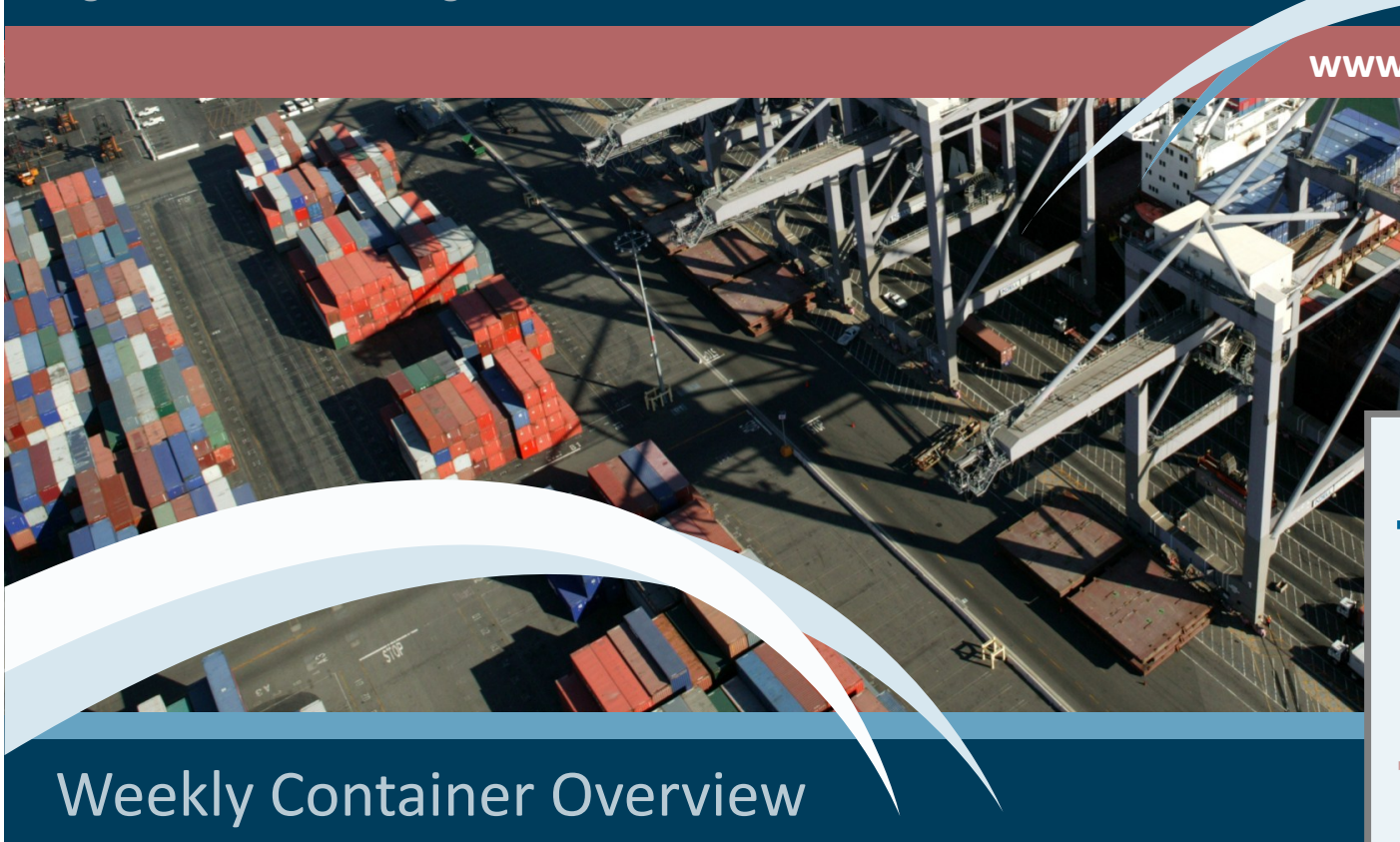

Note: This week's report has been published retroactively due to the government shutdown.

- Ports: The ports of Los Angeles/Long Beach, New York, Norfolk, and Oakland are expected to have the most available containers among the reported port locations over the next 3 weeks. Availability estimates increased from the previous week at Los Angeles/Long Beach, Norfolk, and Oakland, but decreased in New York.

- Inland Locations: Among the reported inland locations, Dallas, Chicago, Memphis, and Columbus are expected to have the most containers available over the next 3 weeks. Availability estimates increased this week in Chicago and Dallas, but decreased in Memphis and Columbus.

- Equipment: Overall, current week availability for all container types combined decreased slightly, down 99 units from the previous week.

- Availability estimates decreased at 10 of the 18 reported locations this weekparticularly in Houston and Tacoma. Other locations saw increases such as New Orleans, Chicago, and Los Angeles/Long Beach particularly for $40 \mathrm{ft}$ highcube containers.

- Availability estimates fell for $20 \mathrm{ft}$ dry containers and both $20 \mathrm{ft}$ and $40 \mathrm{ft}$ refrigerated containers. Estimates for $40 \mathrm{ft}$ dry and high-cube containers increased slightly. See table below.

\begin{tabular}{|l|r|r|r|r|c|}
\hline \multicolumn{6}{|c|}{ Week-to-Week Comparison of Current Week Availability Estimates } \\
\hline \multicolumn{1}{|c|}{ Container type } & $\begin{array}{r}\text { Week of } \\
\text { Sep 18-24 }\end{array}$ & $\begin{array}{c}\text { Week of Sep } \\
\text { 25-Oct 1 }\end{array}$ & $\begin{array}{c}\text { Week of } \\
\text { Oct 2-8 }\end{array}$ & $\begin{array}{c}\text { Week of } \\
\text { Oct 9-15 }\end{array}$ & $\begin{array}{c}\text { \% Change from } \\
\text { Previous Week }\end{array}$ \\
\hline 20ft dry & 9,187 & 10,202 & 7,892 & 7,526 & $-5 \%$ \\
\hline 40ft dry & 8,583 & 9,492 & 8,626 & 8,634 & $0.1 \%$ \\
\hline 40ft high-cube & 19,895 & 19,716 & 18,636 & 19,471 & \multicolumn{1}{c|}{$4 \%$} \\
\hline 40ft refrigerated & 3,815 & 3,903 & 3,828 & 3,306 & $-14 \%$ \\
\hline 20ft refrigerated & 241 & 237 & 260 & 206 & $-21 \%$ \\
\hline
\end{tabular}

Container Availability by Location:

National Overview Maps P. 2-4

Charleston, SC P.5

Chicago, IL P.6

Cincinnati, $\mathrm{OH}$ P.7

Columbus, $\mathrm{OH}$ P.8

Dallas, TX P.9

Denver, CO P.10

Houston, TX P.11

Kansas City, MO P.12

Los Angeles and Long Beach, CA P.13

Memphis, TN P.14

Minneapolis, MN P.15

New Orleans, LA P.16

New York, NY P.17

Norfolk, VA P.18

Oakland, CA P.19

Savannah, GA P.20

Seattle, WA P.21

Tacoma, WA P.22

Understanding this Report P.23

To subscribe to OSCAR, e-mail: April.Taylor@ams.usda.gov 


\section{National Overview}

\section{Week of Oct 9-15, 2013}

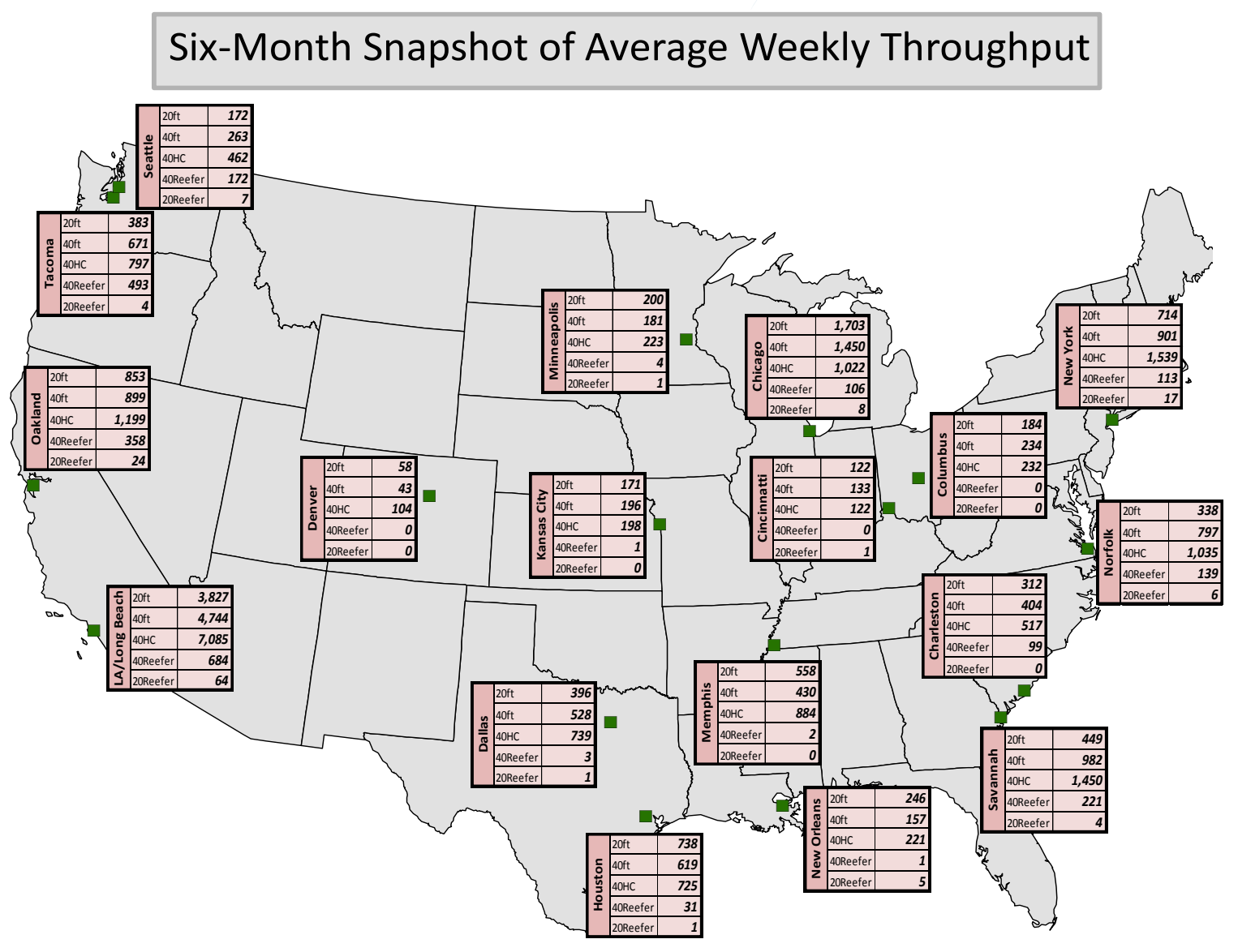

\section{Average Weekly Availability for 20ft Dry Containers}

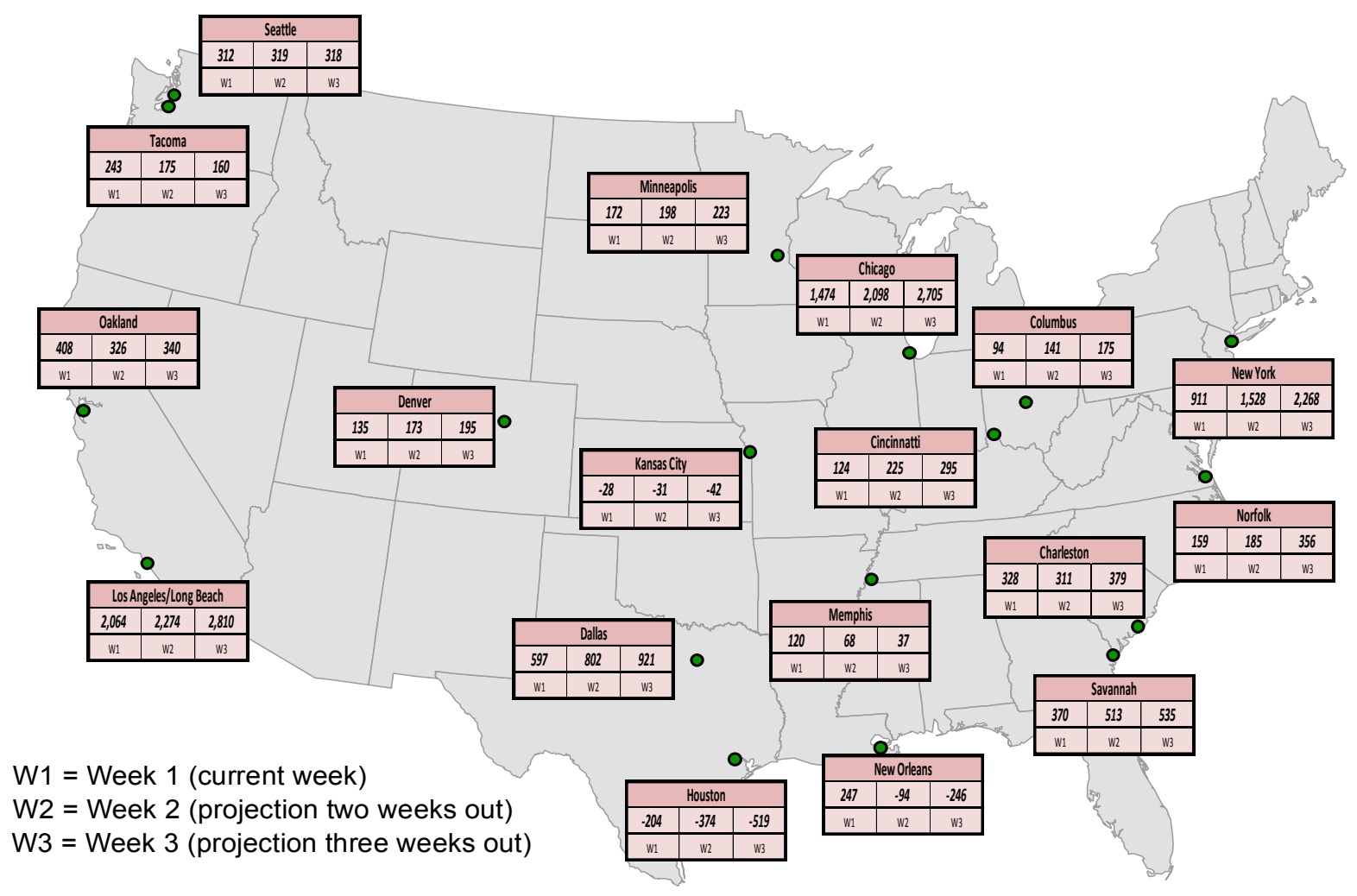




\section{National Overview}

\section{Week of Oct 9-15, 2013}

\section{Average Weekly Availability for 40ft Dry Containers}

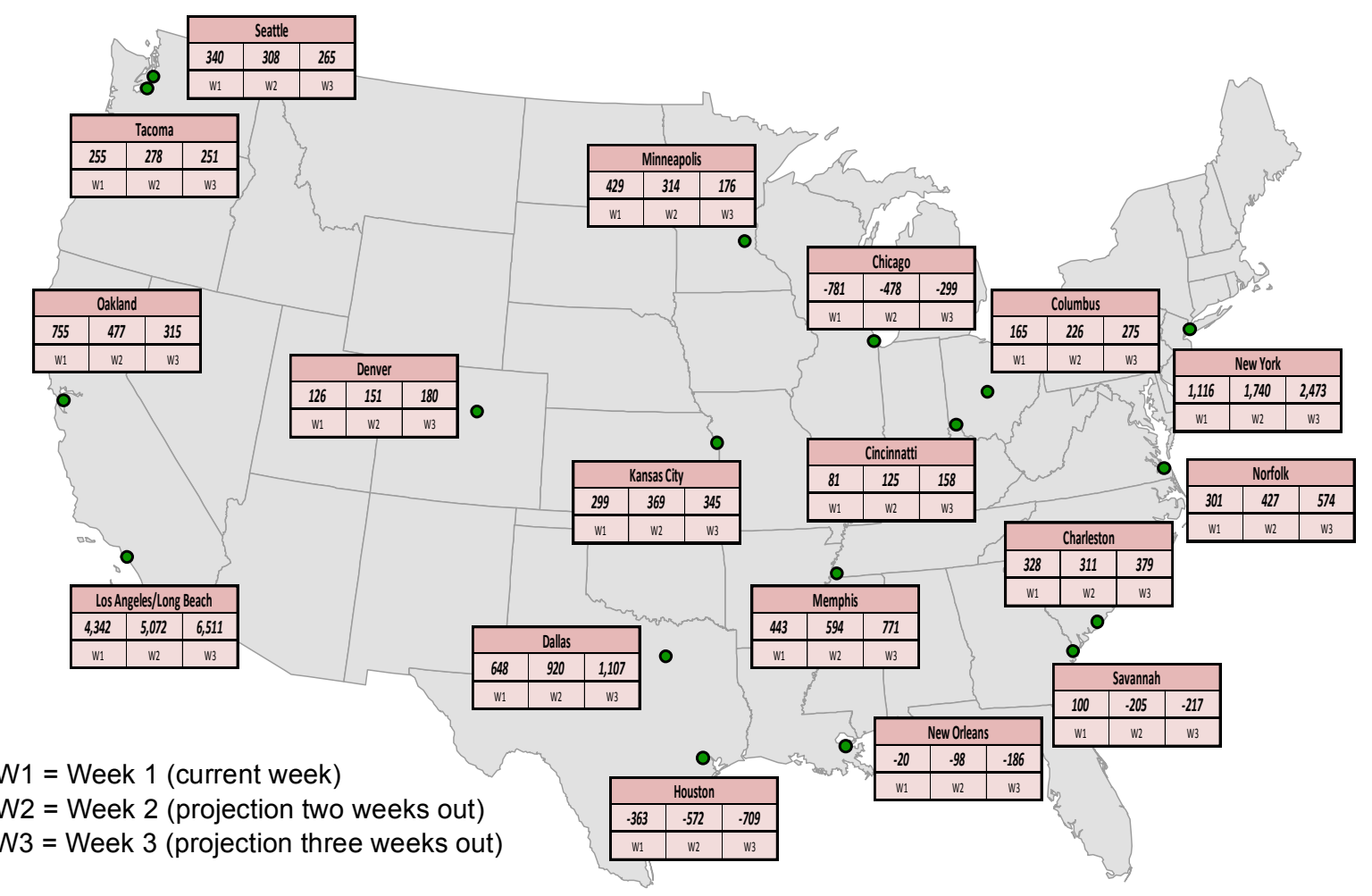

\section{Average Weekly Availability for $40 \mathrm{ft}$ High Cube Containers}

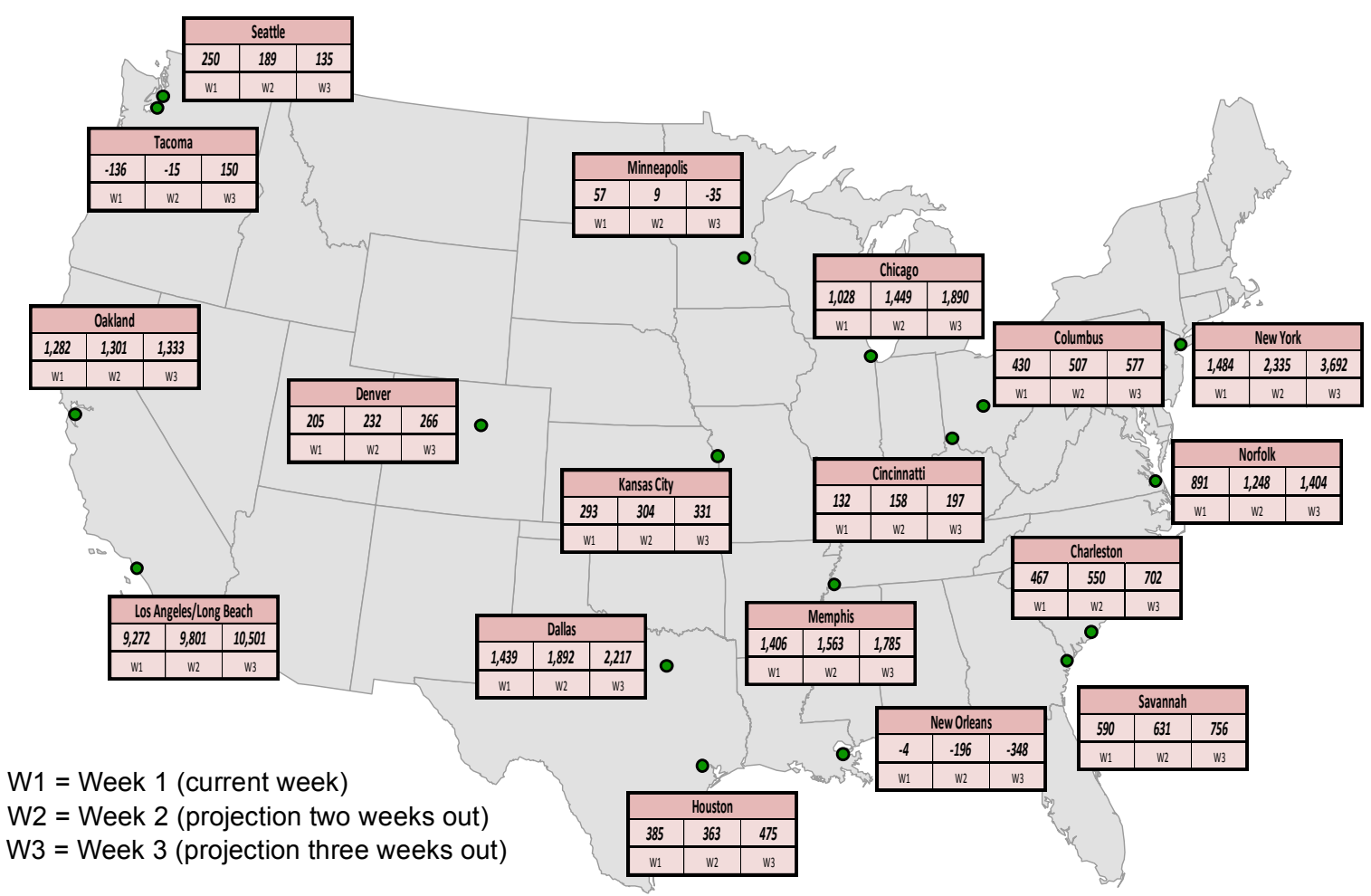




\section{National Overview}

\section{Week of Oct 9-15, 2013}

Average Weekly Availability for 40ft Refrigerated Containers

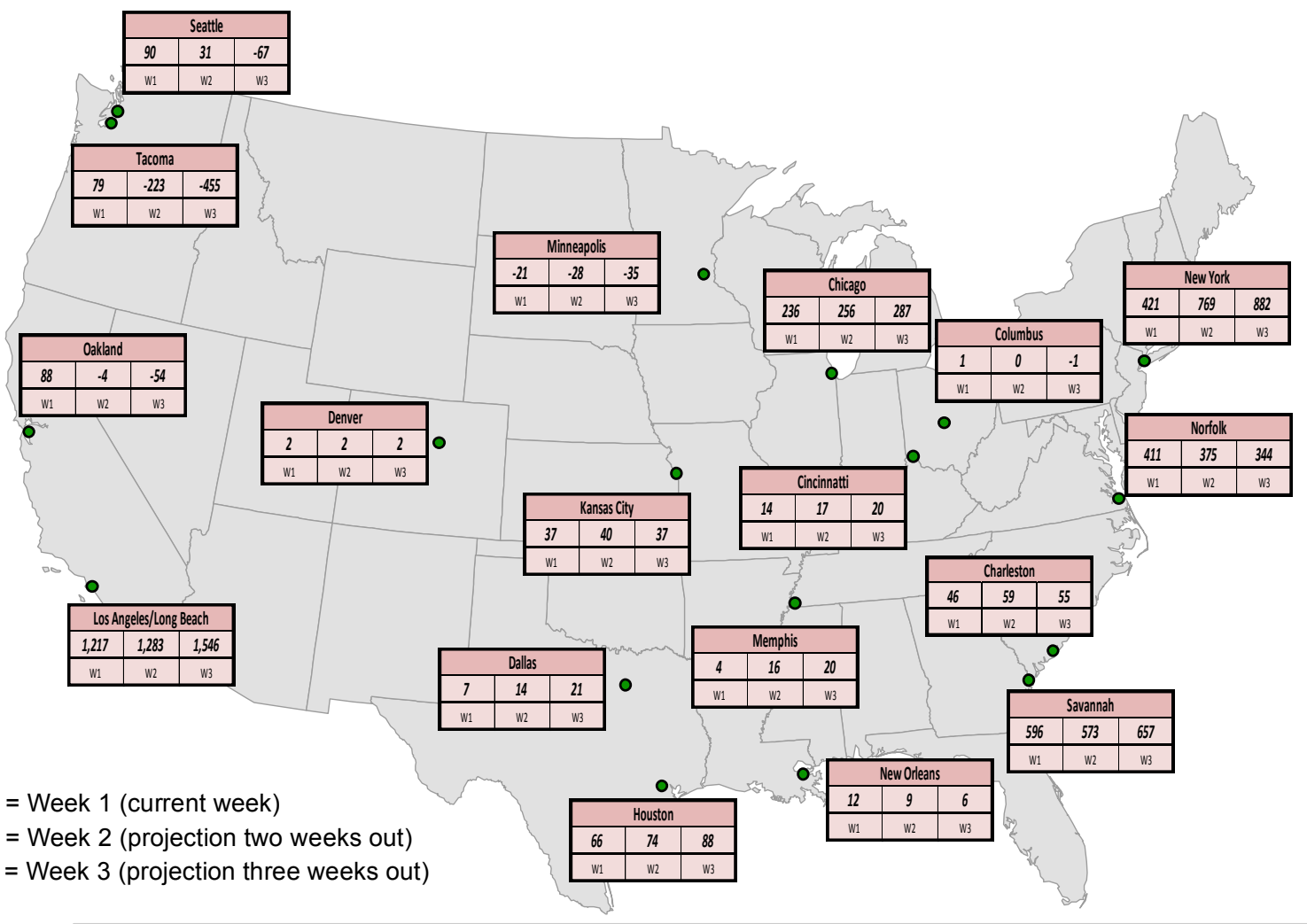

Average Weekly Availability for $20 \mathrm{ft}$ Refrigerated Containers

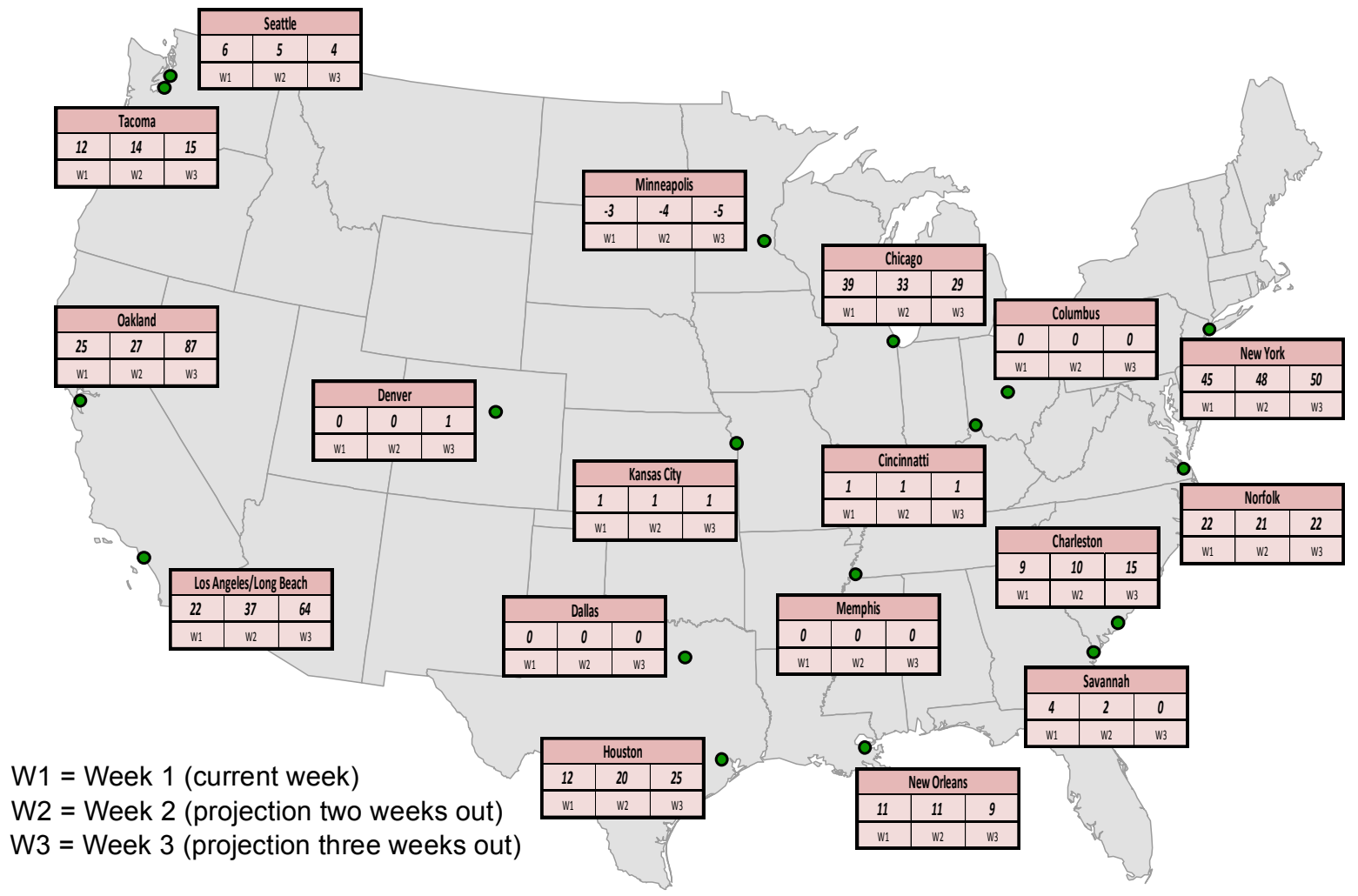




\section{Charleston, SC}

\begin{tabular}{|c|c|c|c|c|c|c|c|c|c|c|c|}
\hline \multicolumn{12}{|c|}{ Charleston } \\
\hline \multicolumn{12}{|c|}{ 20ft Dry } \\
\hline & \multicolumn{10}{|c|}{ Carrier Availability Responses } & Total \\
\hline Current Week: Oct 9-15 & 128 & 119 & 50 & 20 & 10 & 1 & . & . & . & . & 328 \\
\hline Week 2: Oct 16-22 & 125 & 120 & 50 & 24 & -9 & 1 & . & . & . & . & 311 \\
\hline Week 3: Oct 23-29 & 112 & 152 & 50 & 51 & 12 & 2 & . & . & . & . & 379 \\
\hline \multicolumn{12}{|c|}{ 40ft Dry } \\
\hline & \multicolumn{10}{|c|}{ Carrier Availability Responses } & Total \\
\hline Current Week: Oct 9-15 & 161 & 159 & 52 & 20 & 4 & 2 & . & . & . & . & 398 \\
\hline Week 2: Oct 16-22 & 177 & 136 & 80 & 20 & 19 & 5 & . & . & . & . & 437 \\
\hline Week 3: Oct 23-29 & 160 & 172 & 114 & 20 & 33 & 7 & . & . & . & . & 506 \\
\hline \multicolumn{12}{|c|}{ 40ft High Cube } \\
\hline & \multicolumn{10}{|c|}{ Carrier Availability Category Responses } & Total \\
\hline Current Week: Oct 9-15 & 263 & 108 & 40 & 31 & 24 & 1 & . & . & . & . & 467 \\
\hline Week 2: Oct 16-22 & 342 & 89 & 40 & 34 & 43 & 2 & . & . & . & . & 550 \\
\hline Week 3: Oct 23-29 & 423 & 132 & 40 & 70 & 35 & 2 & . & . & . & . & 702 \\
\hline \multicolumn{12}{|c|}{ 40ft Refrigerated } \\
\hline & \multicolumn{10}{|c|}{ Carrier Availability Category Responses } & Total \\
\hline Current Week: Oct 9-15 & 70 & 30 & 16 & 5 & 1 & -76 & . & . & . & . & 46 \\
\hline Week 2: Oct 16-22 & 75 & 30 & 19 & 5 & 1 & -71 & . & . & . & . & 59 \\
\hline Week 3: Oct 23-29 & 80 & 30 & 22 & 5 & 1 & -83 & . & . & . & . & 55 \\
\hline \multicolumn{12}{|c|}{ 20ft Refrigerated } \\
\hline & \multicolumn{10}{|c|}{ Carrier Availability Category Responses } & Total \\
\hline Current Week: Oct 9-15 & 5 & 4 & 0 & . & . & . & . & . & . & . & 9 \\
\hline Week 2: Oct 16-22 & 6 & 4 & 0 & . & . & . & . & . & . & . & 10 \\
\hline Week 3: Oct 23-29 & 7 & 8 & 0 & . & . & . & . & . & . & . & 15 \\
\hline
\end{tabular}

Note: "." denotes no response from carriers.

\section{Six-Month Snapshot of Average Weekly}

\section{Throughput}

June 2012-November 2012

\begin{tabular}{|l|c|c|}
\hline Container Types & $\begin{array}{c}\text { Average Total } \\
\text { Weekly Throughput* }\end{array}$ & $\begin{array}{c}\text { Range of Individual } \\
\text { Carrier Responses }\end{array}$ \\
\hline 20ft & 312 & $0 \ldots 108$ \\
40ft & 404 & $0 \ldots 200$ \\
40ft High Cubes & 517 & $17 \ldots 200$ \\
40ft Refrigerated & 99 & $0 \ldots 60$ \\
20ft Refrigerated & 0 & $0 . .0$ \\
\hline
\end{tabular}

*Data reflect the sum of the individual carriers' average weekly throughput over a 6month timeframe 


\section{Chicago, IL}

\begin{tabular}{|c|c|c|c|c|c|c|c|c|c|c|}
\hline \multicolumn{11}{|c|}{ Chicago } \\
\hline \multicolumn{11}{|c|}{$20 \mathrm{ft}$ Dry } \\
\hline & \multicolumn{9}{|c|}{ Carrier Availability Category Responses } & Total \\
\hline Current Week: Oct 9-15 & 1,043 & 171 & 164 & 100 & 8 & -12 & . & . & . & 1,474 \\
\hline Week 2: Oct 16-22 & 1,428 & 222 & 349 & 100 & 8 & -9 & . & . & . & 2,098 \\
\hline Week 3: Oct 23-29 & 1,824 & 444 & 334 & 100 & 8 & -5 & . & . & . & 2,705 \\
\hline \multicolumn{11}{|c|}{ 40ft Dry } \\
\hline & \multicolumn{9}{|c|}{ Carrier Availability Category Responses } & Total \\
\hline Current Week: Oct 9-15 & 107 & 70 & 8 & -23 & -143 & -800 & . &. & . & -781 \\
\hline Week 2: Oct 16-22 & 177 & 176 & 10 & -5 & -36 & -800 & . & . & . & -478 \\
\hline Week 3: Oct 23-29 & 166 & 281 & 8 & -3 & 49 & -800 & . & . & . & -299 \\
\hline \multicolumn{11}{|c|}{ 40ft High Cube } \\
\hline & \multicolumn{9}{|c|}{ Carrier Availability Category Responses } & Total \\
\hline Current Week: Oct 9-15 & 600 & 331 & 226 & 11 & -11 & -129 & . & . & . & 1,028 \\
\hline Week 2: Oct 16-22 & 600 & 616 & 324 & 10 & -12 & -89 & . & . & . & 1,449 \\
\hline Week 3: Oct 23-29 & 600 & 875 & 455 & 10 & -10 & -40 & . & . & . & 1,890 \\
\hline \multicolumn{11}{|c|}{ 40ft Refrigerated } \\
\hline & \multicolumn{9}{|c|}{ Carrier Availability Category Responses } & Total \\
\hline Current Week: Oct 9-15 & 118 & 108 & 10 & 0 & . & . & . & . & . & 236 \\
\hline Week 2: Oct 16-22 & 135 & 111 & 10 & 0 & . & . & . & . & . & 256 \\
\hline Week 3: Oct 23-29 & 149 & 128 & 10 & 0 & . & . & . & . & . & 287 \\
\hline \multicolumn{11}{|c|}{ 20ft Refrigerated } \\
\hline & \multicolumn{9}{|c|}{ Carrier Availability Category Responses } & Total \\
\hline Current Week: Oct 9-15 & 28 & 11 & . & . & . & . & . & . & . & 39 \\
\hline Week 2: Oct 16-22 & 25 & 8 & . & . & . & . & . & . & . & 33 \\
\hline Week 3: Oct 23-29 & 22 & 7 & 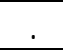 & & . & . & . & . & . & 29 \\
\hline
\end{tabular}

Note: "." denotes no response from carriers.

\begin{tabular}{|l|c|c|}
\hline \multicolumn{3}{|c|}{ Six-Month Snapshot of Average Weekly } \\
Throughput \\
June 2012-November 2012 \\
\hline Container Types & $\begin{array}{c}\text { Average Total } \\
\text { Weekly Throughput* }\end{array}$ & $\begin{array}{c}\text { Range of Individual } \\
\text { Carrier Responses }\end{array}$ \\
\hline 20ft & 1,703 & $93 . .600$ \\
40ft & 1,450 & $5 \ldots 302$ \\
40ft High Cubes & 1,022 & $34 \ldots 405$ \\
40ft Refrigerated & 106 & $0 . .51$ \\
20ft Refrigerated & 8 & $0 . . .4$ \\
\hline
\end{tabular}

*Data reflect the sum of the individual carriers' average weekly throughput over a 6month timeframe 


\section{Cincinnati, $\mathrm{OH}$}

\begin{tabular}{|c|c|c|c|c|c|c|c|c|c|c|}
\hline \multicolumn{11}{|c|}{ Cincinnatti } \\
\hline \multicolumn{11}{|c|}{ 20ft Dry } \\
\hline & \multicolumn{9}{|c|}{ Carrier Availability Category Responses } & Total \\
\hline Current Week: Oct 9-15 & 81 & 20 & 14 & 9 & 6 & -6 & . & . & . & 124 \\
\hline Week 2: Oct 16-22 & 117 & 20 & 12 & 31 & 44 & 1 & . & . & . & 225 \\
\hline Week 3: Oct 23-29 & 155 & 20 & 18 & 34 & 68 & 0 & . & . & . & 295 \\
\hline \multicolumn{11}{|c|}{ 40ft Dry } \\
\hline & \multicolumn{9}{|c|}{ Carrier Availability Category Responses } & Total \\
\hline Current Week: Oct 9-15 & 30 & 19 & 14 & 12 & 10 & -4 & . & . & . & 81 \\
\hline Week 2: Oct 16-22 & 30 & 42 & 14 & 26 & 14 & -1 & . & . & . & 125 \\
\hline Week 3: Oct 23-29 & 30 & 53 & 16 & 41 & 21 & -3 & . & . & . & 158 \\
\hline \multicolumn{11}{|c|}{ 40ft High Cube } \\
\hline & \multicolumn{9}{|c|}{ Carrier Availability Category Responses } & Total \\
\hline Current Week: Oct 9-15 & 62 & 50 & 18 & 11 & -2 & -7 & . & . & . & 132 \\
\hline Week 2: Oct 16-22 & 85 & 50 & 14 & 9 & -1 & 1 & . & . & . & 158 \\
\hline Week 3: Oct 23-29 & 109 & 50 & 14 & 17 & -2 & 9 & . & . & . & 197 \\
\hline \multicolumn{11}{|c|}{ 40ft Refrigerated } \\
\hline & \multicolumn{9}{|c|}{ Carrier Availability Category Responses } & Total \\
\hline Current Week: Oct 9-15 & 14 & 0 & . & & . & . & . & . & . & 14 \\
\hline Week 2: Oct 16-22 & 17 & 0 & . & . & . & . & . & . & . & 17 \\
\hline Week 3: Oct 23-29 & 20 & 0 & . & & . & . & . & . & . & 20 \\
\hline \multicolumn{11}{|c|}{ 20ft Refrigerated } \\
\hline & \multicolumn{9}{|c|}{ Carrier Availability Category Responses } & Total \\
\hline Current Week: Oct 9-15 & 1 & . & . & . & . & . & . & . & . & 1 \\
\hline Week 2: Oct 16-22 & 1 & . & . & . & . & . & . & . & . & 1 \\
\hline Week 3: Oct 23-29 & 1 & . & & & . & . & . & . &. & 1 \\
\hline
\end{tabular}

Note: "." denotes no response from carriers.

\begin{tabular}{|l|c|c|}
\hline \multicolumn{3}{|c|}{ Six-Month Snapshot of Average Weekly } \\
Throughput \\
June 2012-November 2012 \\
\hline Container Types & $\begin{array}{c}\text { Average Total } \\
\text { Weekly Throughput* }\end{array}$ & $\begin{array}{c}\text { Range of Individual } \\
\text { Carrier Responses }\end{array}$ \\
\hline 20ft & 122 & $7 \ldots 35$ \\
40ft & 133 & $8 \ldots 30$ \\
40ft High Cubes & 122 & $11 \ldots 35$ \\
40ft Refrigerated & 0 & $0 \ldots 0$ \\
20ft Refrigerated & 1 & $0 \ldots 1$ \\
\hline
\end{tabular}

*Data reflect the sum of the individual carriers' average weekly throughput over a 6month timeframe 


\section{Columbus, $\mathrm{OH}$}

\begin{tabular}{|c|c|c|c|c|c|c|c|c|c|c|c|}
\hline \multicolumn{12}{|c|}{ Columbus } \\
\hline \multicolumn{12}{|c|}{$20 \mathrm{ft}$ Dry } \\
\hline & \multicolumn{10}{|c|}{ Carrier Availability Category Responses } & Total \\
\hline Current Week: Oct 9-15 & 48 & 30 & 10 & 4 & 3 & -1 &. & . & . & . & 94 \\
\hline Week 2: Oct 16-22 & 64 & 35 & 12 & 28 & 3 & -1 & . & . & . & . & 141 \\
\hline Week 3: Oct 23-29 & 79 & 43 & 12 & 40 & 3 & -2 & . & . & . & . & 175 \\
\hline \multicolumn{12}{|c|}{ 40ft Dry } \\
\hline & \multicolumn{10}{|c|}{ Carrier Availability Category Responses } & Total \\
\hline Current Week: Oct 9-15 & 83 & 38 & 30 & 11 & 3 & 0 & . & . & . & . & 165 \\
\hline Week 2: Oct 16-22 & 119 & 44 & 30 & 11 & 2 & 20 & . & . & . & . & 226 \\
\hline Week 3: Oct 23-29 & 153 & 54 & 30 & 16 & -1 & 23 & . & . & . & . & 275 \\
\hline \multicolumn{12}{|c|}{ 40ft High Cube } \\
\hline & \multicolumn{10}{|c|}{ Carrier Availability Category Responses } & Total \\
\hline Current Week: Oct 9-15 & 231 & 80 & 62 & 41 & 15 & 1 & . & . & . & . & 430 \\
\hline Week 2: Oct 16-22 & 282 & 80 & 79 & 50 & 15 & 1 & . & . & . & . & 507 \\
\hline Week 3: Oct 23-29 & 329 & 80 & 101 & 55 & 14 & -2 & . & . & . & . & 577 \\
\hline \multicolumn{12}{|c|}{ 40ft Refrigerated } \\
\hline & \multicolumn{10}{|c|}{ Carrier Availability Category Responses } & Total \\
\hline Current Week: Oct 9-15 & 1 & 0 & . & . & & & . & . & . & . & 1 \\
\hline Week 2: Oct 16-22 & 0 & 0 & . & . & & & . & . & . & . & 0 \\
\hline Week 3: Oct 23-29 & -1 & 0 &. & . & . & . & . & . & . & . & -1 \\
\hline \multicolumn{12}{|c|}{ 20ft Refrigerated } \\
\hline & \multicolumn{10}{|c|}{ Carrier Availability Category Responses } & Total \\
\hline Current Week: Oct 9-15 & . & . & . & . & . & . & . & . & . & . & 0 \\
\hline Week 2: Oct 16-22 & . & . & . & . & . & . & . & . & . & . & 0 \\
\hline Week 3: Oct 23-29 & . & . & . & . & . & . & . & . & . & . & 0 \\
\hline
\end{tabular}

Note: "." denotes no response from carriers.

\begin{tabular}{|l|c|c|}
\hline \multicolumn{3}{|c|}{ Six-Month Snapshot of Average Weekly } \\
Throughput \\
June 2012-November 2012 \\
\hline Container Types & $\begin{array}{c}\text { Average Total } \\
\text { Weekly Throughput* }\end{array}$ & $\begin{array}{c}\text { Range of Individual } \\
\text { Carrier Responses }\end{array}$ \\
\hline $\mathbf{2 0 f t}$ & 184 & $8 \ldots 60$ \\
40ft & 234 & $6 \ldots 80$ \\
40ft High Cubes & 232 & $15 \ldots 60$ \\
40ft Refrigerated & 0 & $0 \ldots 0$ \\
20ft Refrigerated & 0 & $0 . . .0$ \\
\hline
\end{tabular}

*Data reflect the sum of the individual carriers' average weekly throughput over a 6month timeframe 


\section{Dallas, TX}

\section{Dallas}

$20 \mathrm{ft}$ Dry

\begin{tabular}{|l|c|c|c|c|c|c|c|c|c|c|c|}
\hline & \multicolumn{10}{|c|}{ Carrier Availability Category Responses } & Total \\
\hline Current Week: Oct 9-15 & 251 & 134 & 109 & 83 & 10 & 10 &. &. &. &. & 597 \\
\hline Week 2: Oct 16-22 & 372 & 183 & 97 & 130 & 10 & 10 &. &. &. &. & 802 \\
\hline Week 3: Oct 23-29 & 492 & 141 & 82 & 181 & 10 & 15 &. &. &. &. & 921 \\
\hline \multicolumn{10}{|c|}{ 40ft Dry } \\
\hline
\end{tabular}

Carrier Availability Category Responses

Total

\begin{tabular}{|l|c|c|c|c|c|c|c|c|c|c|c|}
\hline Current Week: Oct 9-15 & 200 & 196 & 190 & 47 & 10 & 5 &. &. &. &. & 648 \\
\hline Week 2: Oct 16-22 & 333 & 259 & 184 & 131 & 8 & 5 &. &. &. &. & 920 \\
\hline Week 3: Oct 23-29 & 461 & 287 & 178 & 165 & 11 & 5 &. &. &. &. & 1,107 \\
\hline \multicolumn{10}{|c|}{ 40ft High Cube }
\end{tabular}

Carrier Availability Category Responses

Total

Current Week: Oct 9-15

Week 2: Oct 16-22

Week 3: Oct 23-29

\begin{tabular}{|l|l|l|l|l|l|l|l|l|l|l|}
\hline 336 & 304 & 300 & 281 & 207 & 11 &. &. &. &. & 1,439 \\
\hline 513 & 461 & 300 & 293 & 315 & 10 &. &. &. &. & 1,892 \\
\hline 680 & 546 & 300 & 270 & 410 & 11 &. &. &. &. & 2,217 \\
\hline
\end{tabular}

40ft Refrigerated

\begin{tabular}{|l|c|c|c|c|c|c|c|c|c|c|c|}
\hline & \multicolumn{1}{|c|}{ Carrier Availability Category Responses } & Total \\
\hline Current Week: Oct 9-15 & 12 & 0 & -5 &. &. &. &. &. &. &. & 7 \\
\hline Week 2: Oct 16-22 & 19 & 0 & -5 &. &. &. &. &. &. &. & 14 \\
\hline Week 3: Oct 23-29 & 26 & 0 & -5 &. &. &. &. &. &. &. & 21 \\
\hline \multicolumn{10}{|c|}{ 20ft Refrigerated } \\
\hline & \multicolumn{10}{|c|}{ Carrier Availability Category Responses } & Total \\
\hline Current Week: Oct 9-15 & 0 &. &. &. &. &. &. &. &. &. & 0 \\
\hline Week 2: Oct 16-22 & 0 &. &. &. &. &. &. &. &. &. & 0 \\
\hline Week 3: Oct 23-29 & 0 &. &. &. &. &. &. &. &. &. & 0 \\
\hline
\end{tabular}

Note: "." denotes no response from carriers.

\section{Six-Month Snapshot of Average Weekly} Throughput

June 2012-Novem ber 2012

\begin{tabular}{|l|c|c|}
\hline Container Types & $\begin{array}{c}\text { Average Total } \\
\text { Weekly Throughput* }\end{array}$ & $\begin{array}{c}\text { Range of Individual } \\
\text { Carrier Responses }\end{array}$ \\
\hline 20ft & 396 & $5 \ldots 150$ \\
40ft & 528 & $12 \ldots .250$ \\
40ft High Cubes & 739 & $22 \ldots 250$ \\
40ft Refrigerated & 3 & $0 \ldots 3$ \\
20ft Refrigerated & 1 & $0 . .1$ \\
\hline
\end{tabular}

*Data reflect the sum of the individual carriers' average weekly throughput over a 6month timeframe 


\section{Denver, CO}

\begin{tabular}{|c|c|c|c|c|c|c|c|c|c|c|c|}
\hline \multicolumn{12}{|c|}{ Denver } \\
\hline \multicolumn{12}{|c|}{ 20ft Dry } \\
\hline & \multicolumn{10}{|c|}{ Carrier Availability Category Responses } & Total \\
\hline Current Week: Oct 9-15 & 152 & 5 & 1 & 0 & -5 & -18 & . & . & . & . & 135 \\
\hline Week 2: Oct 16-22 & 174 & 6 & 1 & -1 & -7 & 0 & . & . & . & . & 173 \\
\hline Week 3: Oct 23-29 & 197 & 6 & 1 & -1 & -8 & 0 & . & . & . & . & 195 \\
\hline \multicolumn{12}{|c|}{ 40ft Dry } \\
\hline & \multicolumn{10}{|c|}{ Carrier Availability Category Responses } & Total \\
\hline Current Week: Oct 9-15 & 97 & 8 & 8 & 6 & 4 & 3 & . & . & . & . & 126 \\
\hline Week 2: Oct 16-22 & 117 & 9 & 12 & 5 & 5 & 3 & . & . & . & . & 151 \\
\hline Week 3: Oct 23-29 & 135 & 11 & 10 & 17 & 4 & 3 & . & . & . & . & 180 \\
\hline \multicolumn{12}{|c|}{ 40ft High Cube } \\
\hline & \multicolumn{10}{|c|}{ Carrier Availability Category Responses } & Total \\
\hline Current Week: Oct 9-15 & 116 & 70 & 9 & 9 & 3 & -2 & . & . & . & . & 205 \\
\hline Week 2: Oct 16-22 & 144 & 70 & 6 & 9 & 4 & -1 & . & . & . & . & 232 \\
\hline Week 3: Oct 23-29 & 170 & 70 & 11 & 10 & 2 & 3 & . & . & . & . & 266 \\
\hline \multicolumn{12}{|c|}{ 40ft Refrigerated } \\
\hline & \multicolumn{10}{|c|}{ Carrier Availability Category Responses } & Total \\
\hline Current Week: Oct 9-15 & 2 & 0 & . & . & & & . & . & . & . & 2 \\
\hline Week 2: Oct 16-22 & 2 & 0 & . & . & & & . & . & . & . & 2 \\
\hline Week 3: Oct 23-29 & 2 & 0 & . & . & 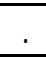 & . & . & . & . & . & 2 \\
\hline \multicolumn{12}{|c|}{ 20ft Refrigerated } \\
\hline & \multicolumn{10}{|c|}{ Carrier Availability Category Responses } & Total \\
\hline Current Week: Oct 9-15 & 0 & . & . & . & & & . & . & . & . & 0 \\
\hline Week 2: Oct 16-22 & 0 & . & . & . & & & . & . & . & . & 0 \\
\hline Week 3: Oct 23-29 & 1 & . & . & . & . & & . & . & . & . & 1 \\
\hline
\end{tabular}

Note: "." denotes no response from carriers.

\section{Six-Month Snapshot of Average Weekly Throughput \\ June 2012-November 2012}

\begin{tabular}{|l|c|c|}
\hline Container Types & $\begin{array}{c}\text { Average Total } \\
\text { Weekly Throughput* }\end{array}$ & $\begin{array}{c}\text { Range of Individual } \\
\text { Carrier Responses }\end{array}$ \\
\hline 20ft & 58 & $3 \ldots 17$ \\
40ft & 43 & $1 \ldots 10$ \\
40ft High Cubes & 104 & $1 \ldots . .50$ \\
40ft Refrigerated & 0 & $0 . . .0$ \\
20ft Refrigerated & 0 & $0 . . .0$ \\
\hline
\end{tabular}

*Data reflect the sum of the individual carriers' average weekly throughput over a 6month timeframe 


\section{Houston, TX}

\begin{tabular}{|c|c|c|c|c|c|c|c|c|c|c|c|}
\hline \multicolumn{12}{|c|}{ Houston } \\
\hline \multicolumn{12}{|c|}{ 20ft Dry } \\
\hline & \multicolumn{10}{|c|}{ Carrier Availability Category Responses } & Total \\
\hline Current Week: Oct 9-15 & 150 & 50 & 40 & 8 & -52 & -400 & . & . & . & . & -204 \\
\hline Week 2: Oct 16-22 & 150 & 8 & 43 & 9 & -40 & -544 & . & . & . & . & -374 \\
\hline Week 3: Oct 23-29 & 150 & 5 & 44 & 9 & -27 & -700 & . & . & . & . & -519 \\
\hline \multicolumn{12}{|c|}{ 40ft Dry } \\
\hline & \multicolumn{10}{|c|}{ Carrier Availability Category Responses } & Total \\
\hline Current Week: Oct 9-15 & 70 & 66 & 47 & 1 & -35 & -512 & . & . & . & . & -363 \\
\hline Week 2: Oct 16-22 & 70 & 43 & 15 & 2 & -45 & -657 & . & . & . & . & -572 \\
\hline Week 3: Oct 23-29 & 70 & 77 & 10 & 4 & -60 & -810 & . & . & . & . & -709 \\
\hline \multicolumn{12}{|c|}{ 40ft High Cube } \\
\hline & \multicolumn{10}{|c|}{ Carrier Availability Category Responses } & Total \\
\hline Current Week: Oct 9-15 & 500 & 105 & 98 & 5 & 2 & -325 & . &. & . & & 385 \\
\hline Week 2: Oct 16-22 & 500 & 137 & 89 & 2 & -15 & -350 & . & . & . & . & 363 \\
\hline Week 3: Oct 23-29 & 500 & 214 & 149 & 1 & -20 & -369 & . & . & . & & 475 \\
\hline \multicolumn{12}{|c|}{ 40ft Refrigerated } \\
\hline & \multicolumn{10}{|c|}{ Carrier Availability Category Responses } & Total \\
\hline Current Week: Oct 9-15 & 30 & 26 & 8 & 2 & 0 & . & . & . & . & . & 66 \\
\hline Week 2: Oct 16-22 & 25 & 40 & 7 & 2 & 0 & . & . & . & . & . & 74 \\
\hline Week 3: Oct 23-29 & 20 & 58 & 8 & 2 & 0 & . & . & . & . & . & 88 \\
\hline \multicolumn{12}{|c|}{ 20ft Refrigerated } \\
\hline & \multicolumn{10}{|c|}{ Carrier Availability Category Responses } & Total \\
\hline Current Week: Oct 9-15 & 15 & 0 & -3 & & & . & . & . & . & & 12 \\
\hline Week 2: Oct 16-22 & 19 & 0 & 1 & & . & . & . & . & . & . & 20 \\
\hline Week 3: Oct 23-29 & 23 & 0 & 2 & . & . & . &. & . &. & . & 25 \\
\hline
\end{tabular}

Note: "." denotes no response from carriers.

\begin{tabular}{|l|c|c|}
\hline \multicolumn{3}{|c|}{ Six-Month Snapshot of Average Weekly } \\
Throughput \\
June 2012-November 2012 \\
\hline Container Types & $\begin{array}{c}\text { Average Total } \\
\text { Weekly Throughput* }\end{array}$ & $\begin{array}{c}\text { Range of Individual } \\
\text { Carrier Responses }\end{array}$ \\
\hline 20ft & 738 & $50 \ldots 316$ \\
40ft & 619 & $50 \ldots 126$ \\
40ft High Cubes & 725 & $26 \ldots 355$ \\
40ft Refrigerated & 31 & $0 . .12$ \\
20ft Refrigerated & 1 & $0 . .1$ \\
\hline
\end{tabular}

*Data reflect the sum of the individual carriers' average weekly throughput over a 6 month timeframe 


\section{Kansas City, MO}

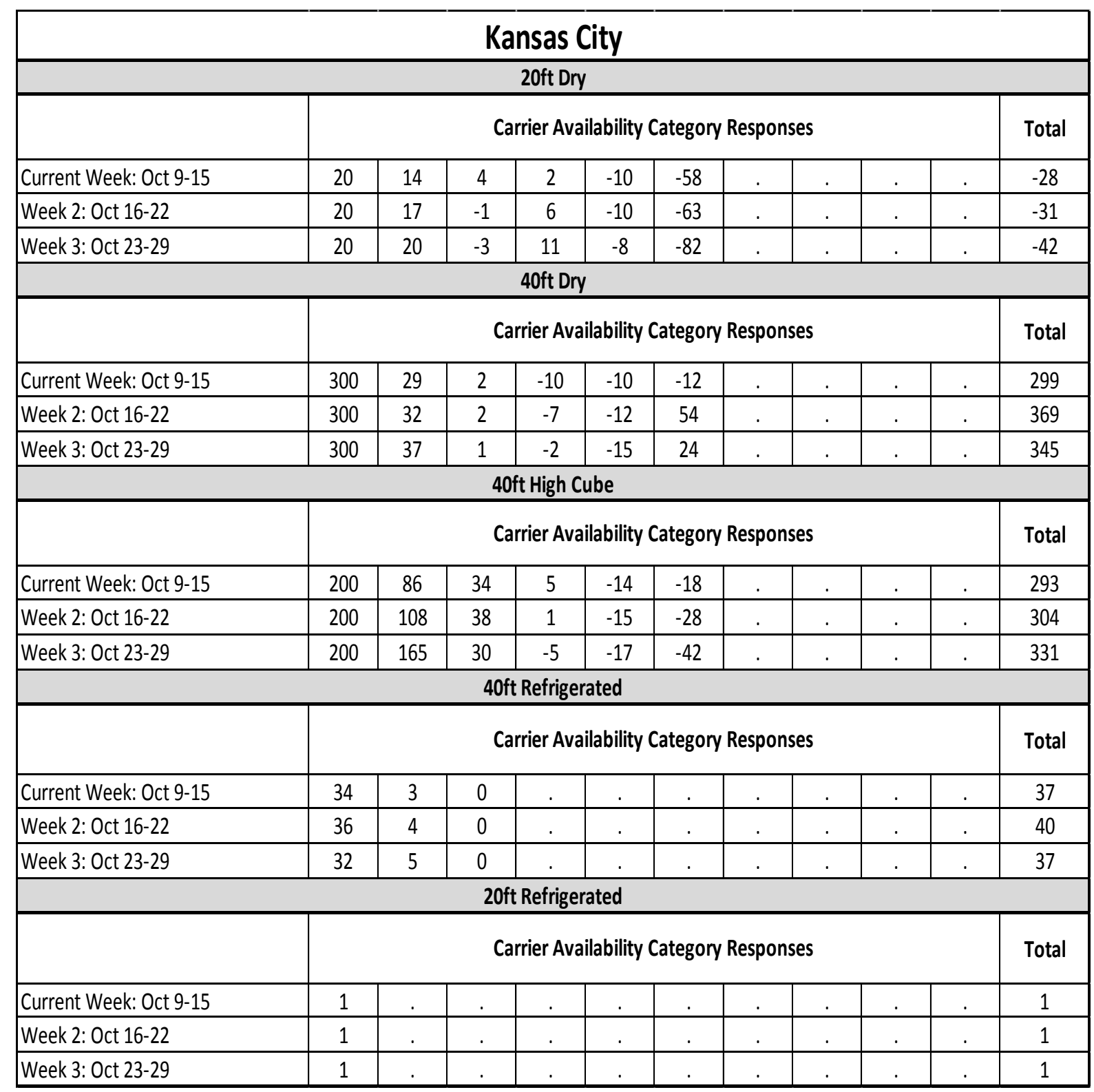

Note: "." denotes no response from carriers.

\begin{tabular}{|l|c|c|}
\hline \multicolumn{3}{|c|}{ Six-Month Snapshot of Average Weekly } \\
Throughput \\
June 2012-November 2012 \\
\hline Container Types & Average Total & Range of Individual \\
& Weekly Throughput* & Carrier Responses \\
\hline 20ft & 171 & $7 . .60$ \\
40ft & 196 & $9 . .75$ \\
40ft High Cubes & 198 & $14 \ldots 59$ \\
40ft Refrigerated & 1 & $0 . . .1$ \\
20ft Refrigerated & 0 & $0 . . .0$ \\
\hline
\end{tabular}

*Data reflect the sum of the individual carriers' average weekly throughput over a 6month timeframe 


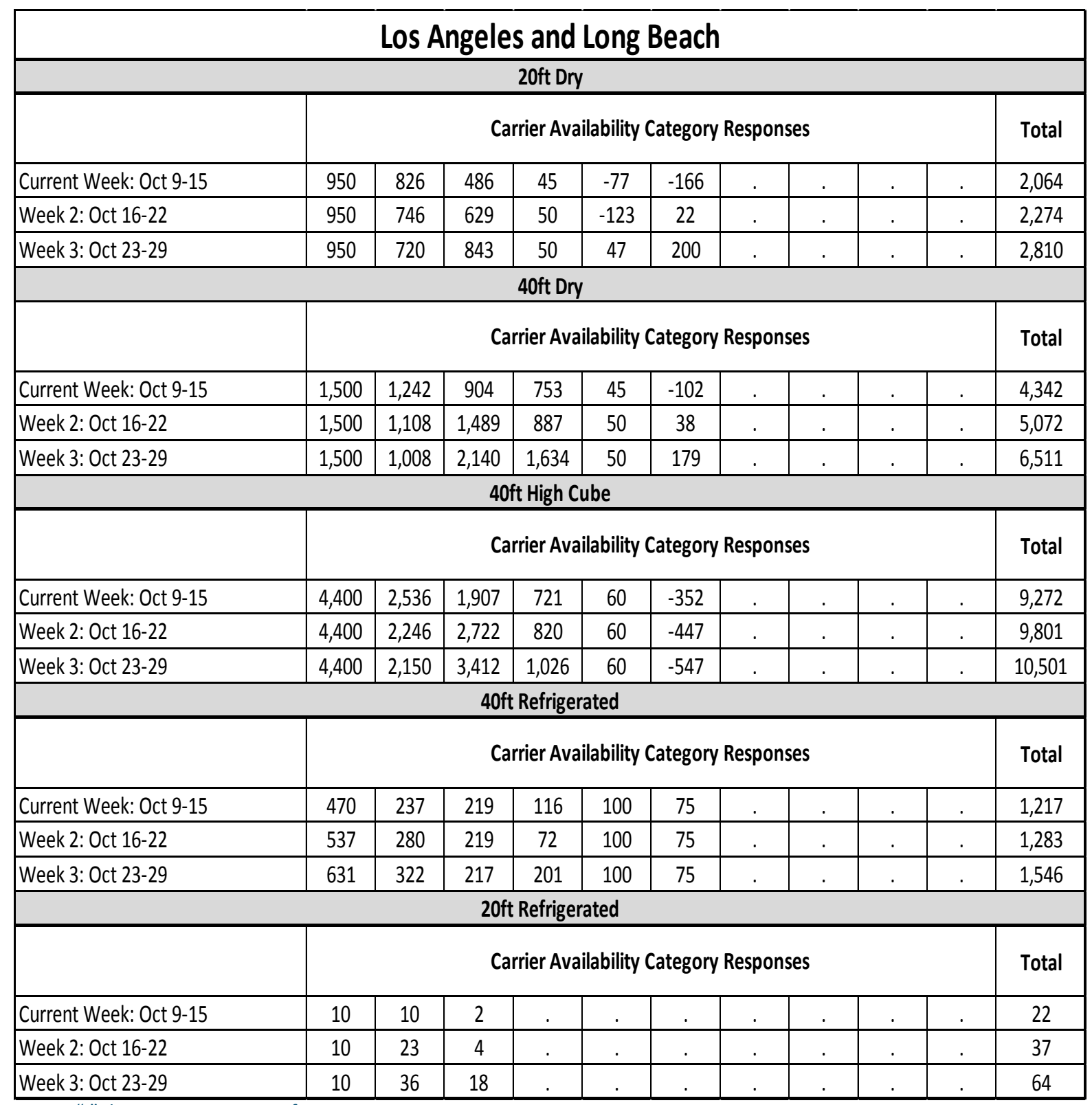

Note: "." denotes no response from carriers.

\begin{tabular}{|l|c|c|}
\hline \multicolumn{3}{|c|}{ Six-Month Snapshot of Average Weekly } \\
Throughput \\
June 2012-November 2012 \\
\hline Container Types & $\begin{array}{c}\text { Average Total } \\
\text { Weekly Throughput* }\end{array}$ & $\begin{array}{c}\text { Range of Individual } \\
\text { Carrier Responses }\end{array}$ \\
\hline $\mathbf{2 0 f t}$ & 3,827 & $46 . .2500$ \\
$\mathbf{4 0 f t}$ & 4,744 & $83 . . .3000$ \\
$\mathbf{4 0 f t}$ High Cubes & 7,085 & $203 \ldots 3300$ \\
$\mathbf{4 0 f t}$ Refrigerated & 684 & $21 \ldots 300$ \\
20ft Refrigerated & 64 & $1 \ldots 50$ \\
\hline
\end{tabular}

*Data reflect the sum of the individual carriers' average weekly throughput over a 6month timeframe 


\section{Memphis, TN}

\begin{tabular}{|c|c|c|c|c|c|c|c|c|c|c|}
\hline \multicolumn{11}{|c|}{ Memphis } \\
\hline \multicolumn{11}{|c|}{$20 f t$ Dry } \\
\hline & \multicolumn{9}{|c|}{ Carrier Availability Category Responses } & Total \\
\hline Current Week: Oct 9-15 & 93 & 90 & 19 & 3 & -34 & -51 & . & . & . & 120 \\
\hline Week 2: Oct 16-22 & 48 & 90 & 19 & 2 & -54 & -37 & . & . & . & 68 \\
\hline Week 3: Oct 23-29 & 55 & 90 & 17 & 2 & -73 & -54 & . & . & . & 37 \\
\hline \multicolumn{11}{|c|}{ 40ft Dry } \\
\hline & \multicolumn{9}{|c|}{ Carrier Availability Category Responses } & Total \\
\hline Current Week: Oct 9-15 & 150 & 130 & 88 & 39 & 32 & 4 & . & . & . & 443 \\
\hline Week 2: Oct 16-22 & 150 & 205 & 121 & 87 & 23 & 8 & . & . & . & 594 \\
\hline Week 3: Oct 23-29 & 150 & 268 & 192 & 131 & 20 & 10 & . & . & . & 771 \\
\hline \multicolumn{11}{|c|}{ 40ft High Cube } \\
\hline & \multicolumn{9}{|c|}{ Carrier Availability Category Responses } & Total \\
\hline Current Week: Oct 9-15 & 1,050 & 148 & 103 & 81 & 13 & 11 & . & . & . & 1,406 \\
\hline Week 2: Oct 16-22 & 1,050 & 171 & 146 & 171 & 17 & 8 & . & . & . & 1,563 \\
\hline Week 3: Oct 23-29 & 1,050 & 236 & 214 & 266 & 11 & 8 & . & . & . & 1,785 \\
\hline \multicolumn{11}{|c|}{ 40ft Refrigerated } \\
\hline & \multicolumn{9}{|c|}{ Carrier Availability Category Responses } & Total \\
\hline Current Week: Oct 9-15 & 3 & 1 & 0 & & . & . & . & . & . & 4 \\
\hline Week 2: Oct 16-22 & 14 & 2 & 0 & . & . & . & . & . & . & 16 \\
\hline Week 3: Oct 23-29 & 17 & 3 & 0 & . & . & . & . & . & . & 20 \\
\hline \multicolumn{11}{|c|}{ 20ft Refrigerated } \\
\hline & \multicolumn{9}{|c|}{ Carrier Availability Category Responses } & Total \\
\hline Current Week: Oct 9-15 & . & . & & & . & . & . & . & . & 0 \\
\hline Week 2: Oct 16-22 & . & . & & . & . & . & . & . & . & 0 \\
\hline Week 3: Oct 23-29 & . & . & & . & . & . & . & . & . & 0 \\
\hline
\end{tabular}

Note: "." denotes no response from carriers.

\begin{tabular}{|l|c|c|}
\hline \multicolumn{3}{|c|}{ Six-Month Snapshot of Average Weekly } \\
Throughput \\
June 2012-November 2012 \\
\hline Container Types & $\begin{array}{c}\text { Average Total } \\
\text { Weekly Throughput* }\end{array}$ & $\begin{array}{c}\text { Range of Individual } \\
\text { Carrier Responses }\end{array}$ \\
\hline $\mathbf{2 0 f t}$ & 558 & $11 \ldots 300$ \\
$\mathbf{4 0 f t}$ & 430 & $11 \ldots 200$ \\
$\mathbf{4 0 f t}$ High Cubes & 884 & $9 \ldots 600$ \\
40ft Refrigerated & 2 & $0 . .2$ \\
20ft Refrigerated & 0 & $0 . . .0$ \\
\hline
\end{tabular}

*Data reflect the sum of the individual carriers' average weekly throughput over a 6month timeframe 


\section{Minneapolis, MN}

\begin{tabular}{|c|c|c|c|c|c|c|c|c|c|c|}
\hline \multicolumn{11}{|c|}{ Minneapolis } \\
\hline \multicolumn{11}{|c|}{$20 f t$ Dry } \\
\hline & \multicolumn{9}{|c|}{ Carrier Availability Category Responses } & Total \\
\hline Current Week: Oct 9-15 & 91 & 67 & 20 & 19 & -8 & -17 & . & . & . & 172 \\
\hline Week 2: Oct 16-22 & 82 & 73 & 20 & 29 & -8 & 2 & . & . & . & 198 \\
\hline Week 3: Oct 23-29 & 80 & 82 & 20 & 45 & -7 & 3 & . & . & . & 223 \\
\hline \multicolumn{11}{|c|}{ 40ft Dry } \\
\hline & \multicolumn{9}{|c|}{ Carrier Availability Category Responses } & Total \\
\hline Current Week: Oct 9-15 & 300 & 79 & 41 & 25 & -2 & -14 & . & . & . & 429 \\
\hline Week 2: Oct 16-22 & 300 & 19 & -15 & 9 & -11 & 12 & . & . & . & 314 \\
\hline Week 3: Oct 23-29 & 300 & -44 & -47 & -32 & -9 & 8 & . & . & . & 176 \\
\hline \multicolumn{11}{|c|}{ 40ft High Cube } \\
\hline & \multicolumn{9}{|c|}{ Carrier Availability Category Responses } & Total \\
\hline Current Week: Oct 9-15 & 70 & 58 & 12 & -8 & -16 & -59 & . & . & . & 57 \\
\hline Week 2: Oct 16-22 & 70 & 62 & 11 & -8 & -24 & -102 & . & . & . & 9 \\
\hline Week 3: Oct 23-29 & 70 & 66 & 7 & -9 & -26 & -143 & . & . & . & -35 \\
\hline \multicolumn{11}{|c|}{ 40ft Refrigerated } \\
\hline & \multicolumn{9}{|c|}{ Carrier Availability Category Responses } & Total \\
\hline Current Week: Oct 9-15 & 0 & -21 & & & . & . & . & . & . & -21 \\
\hline Week 2: Oct 16-22 & 0 & -28 & . & & . & . & . & . & . & -28 \\
\hline Week 3: Oct 23-29 & 0 & -35 &. & & . & . & . & . & . & -35 \\
\hline \multicolumn{11}{|c|}{ 20ft Refrigerated } \\
\hline & \multicolumn{9}{|c|}{ Carrier Availability Category Responses } & Total \\
\hline Current Week: Oct 9-15 & -3 & . & . & & . & . & . & . & . & -3 \\
\hline Week 2: Oct 16-22 & -4 & . & . & . & . & . & . & . & . & -4 \\
\hline Week 3: Oct 23-29 & -5 & . & 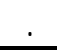 & & . & . & . & . & . & -5 \\
\hline
\end{tabular}

Note: "." denotes no response from carriers.

\section{Six-Month Snapshot of Average Weekly} Throughput

June 2012-November 2012

\begin{tabular}{|l|c|c|}
\hline Container Types & $\begin{array}{c}\text { Average Total } \\
\text { Weekly Throughput* }\end{array}$ & $\begin{array}{c}\text { Range of Individual } \\
\text { Carrier Responses }\end{array}$ \\
\hline 20ft & 200 & $3 \ldots 102$ \\
40ft & 181 & $1 \ldots 80$ \\
40ft High Cubes & 223 & $2 \ldots 144$ \\
40ft Refrigerated & 4 & $0 \ldots 4$ \\
20ft Refrigerated & 1 & $0 \ldots 1$ \\
\hline
\end{tabular}

*Data reflect the sum of the individual carriers' average weekly throughput over a 6month timeframe 


\section{New Orleans, LA}

\begin{tabular}{|c|c|c|c|c|c|c|c|c|c|c|}
\hline \multicolumn{11}{|c|}{ New Orleans } \\
\hline \multicolumn{11}{|c|}{$20 \mathrm{ft}$ Dry } \\
\hline & \multicolumn{9}{|c|}{ Carrier Availability Category Responses } & Total \\
\hline Current Week: Oct 9-15 & 272 & 14 & 5 & 0 & 0 & -44 & . & . & . & 247 \\
\hline Week 2: Oct 16-22 & -10 & 14 & 6 & 0 & 0 & -104 & . & . & . & -94 \\
\hline Week 3: Oct 23-29 & -78 & 10 & 11 & 0 & 1 & -190 & . & . & . & -246 \\
\hline \multicolumn{11}{|c|}{ 40ft Dry } \\
\hline & \multicolumn{9}{|c|}{ Carrier Availability Category Responses } & Total \\
\hline Current Week: Oct 9-15 & 10 & 4 & 0 & 0 & -14 & -20 & . & . & . & -20 \\
\hline Week 2: Oct 16-22 & 14 & 2 & 0 & 1 & -25 & -90 & . & . & . & -98 \\
\hline Week 3: Oct 23-29 & 12 & 3 & 0 & -3 & -36 & -162 & . & . & . & -186 \\
\hline \multicolumn{11}{|c|}{ 40ft High Cube } \\
\hline & \multicolumn{9}{|c|}{ Carrier Availability Category Responses } & Total \\
\hline Current Week: Oct 9-15 & 38 & 14 & 8 & 1 & 0 & -65 & . & . & . & -4 \\
\hline Week 2: Oct 16-22 & 22 & 12 & 14 & 0 & 0 & -244 & . & . & . & -196 \\
\hline Week 3: Oct 23-29 & 13 & 10 & 20 & 0 & 0 & -391 & . & . & . & -348 \\
\hline \multicolumn{11}{|c|}{ 40ft Refrigerated } \\
\hline & \multicolumn{9}{|c|}{ Carrier Availability Category Responses } & Total \\
\hline Current Week: Oct 9-15 & 21 & 0 & -9 & . & . & . & . & . & . & 12 \\
\hline Week 2: Oct 16-22 & 18 & 0 & -9 & . & . & . & . & . & . & 9 \\
\hline Week 3: Oct 23-29 & 15 & 0 & -9 & . & . & . & . & . & . & 6 \\
\hline \multicolumn{11}{|c|}{ 20ft Refrigerated } \\
\hline & \multicolumn{9}{|c|}{ Carrier Availability Category Responses } & Total \\
\hline Current Week: Oct 9-15 & 6 & 5 & . & . & . & . & . & . & . & 11 \\
\hline Week 2: Oct 16-22 & 6 & 5 & . & . & . & . & . & . & . & 11 \\
\hline Week 3: Oct 23-29 & 4 & 5 & . & . & . & . & . & & . & 9 \\
\hline
\end{tabular}

Note: "." denotes no response from carriers.

\begin{tabular}{|l|c|c|}
\hline \multicolumn{3}{|c|}{ Six-Month Snapshot of Average Weekly } \\
Throughput \\
June 2012-November 2012 \\
\hline Container Types & $\begin{array}{c}\text { Average Total } \\
\text { Weekly Throughput* }\end{array}$ & $\begin{array}{c}\text { Range of Individual } \\
\text { Carrier Responses }\end{array}$ \\
\hline 20ft & 246 & $5 \ldots 158$ \\
40ft & 157 & $5 \ldots 80$ \\
40ft High Cubes & 221 & $5 \ldots 139$ \\
40ft Refrigerated & 1 & $0 \ldots 1$ \\
20ft Refrigerated & 5 & $0 . .5$ \\
\hline
\end{tabular}

*Data reflect the sum of the individual carriers' average weekly throughput over a 6month timeframe 


\section{New York, NY}

\begin{tabular}{|c|c|c|c|c|c|c|c|c|c|c|}
\hline \multicolumn{11}{|c|}{ New York } \\
\hline \multicolumn{11}{|c|}{$20 \mathrm{ft}$ Dry } \\
\hline & \multicolumn{9}{|c|}{ Carrier Availability Category Responses } & Total \\
\hline Current Week: Oct 9-15 & 267 & 205 & 168 & 161 & 80 & 30 & . & . & . & 911 \\
\hline Week 2: Oct 16-22 & 532 & 345 & 353 & 178 & 80 & 40 & . & . & . & 1,528 \\
\hline Week 3: Oct 23-29 & 926 & 491 & 558 & 168 & 80 & 45 & . & & . & 2,268 \\
\hline \multicolumn{11}{|c|}{ 40ft Dry } \\
\hline & \multicolumn{9}{|c|}{ Carrier Availability Category Responses } & Total \\
\hline Current Week: Oct 9-15 & 422 & 385 & 200 & 120 & 25 & -36 & . & $\dot{.}$ & . & 1,116 \\
\hline Week 2: Oct 16-22 & 620 & 548 & 370 & 120 & 35 & 47 & . & . & . & 1,740 \\
\hline Week 3: Oct 23-29 & 817 & 848 & 608 & 120 & 40 & 40 & . & & . & 2,473 \\
\hline \multicolumn{11}{|c|}{ 40ft High Cube } \\
\hline & \multicolumn{9}{|c|}{ Carrier Availability Category Responses } & Total \\
\hline Current Week: Oct 9-15 & 607 & 400 & 282 & 125 & 45 & 25 & . & . & . & 1,484 \\
\hline Week 2: Oct 16-22 & 935 & 400 & 593 & 308 & 64 & 35 & . & . & . & 2,335 \\
\hline Week 3: Oct 23-29 & 1,529 & 400 & 992 & 666 & 55 & 50 & . & . & . & 3,692 \\
\hline \multicolumn{11}{|c|}{ 40ft Refrigerated } \\
\hline & \multicolumn{9}{|c|}{ Carrier Availability Category Responses } & Total \\
\hline Current Week: Oct 9-15 & 262 & 126 & 34 & 10 & 5 & -16 & . & & . & 421 \\
\hline Week 2: Oct 16-22 & 373 & 181 & 88 & 10 & 5 & 112 & . & . & . & 769 \\
\hline Week 3: Oct 23-29 & 455 & 231 & 175 & 10 & 5 & 6 & . & . & . & 882 \\
\hline \multicolumn{11}{|c|}{ 20ft Refrigerated } \\
\hline & \multicolumn{9}{|c|}{ Carrier Availability Category Responses } & Total \\
\hline Current Week: Oct 9-15 & 35 & 5 & 5 & . & . & . & . & . & . & 45 \\
\hline Week 2: Oct 16-22 & 40 & 5 & 3 & . & . & . & . & 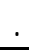 & . & 48 \\
\hline Week 3: Oct 23-29 & 45 & 5 & 0 & . & . & . & . & & $\cdot$ & 50 \\
\hline
\end{tabular}

Note: "." denotes no response from carriers.

\begin{tabular}{|l|c|c|}
\hline \multicolumn{3}{|c|}{ Six-Month Snapshot of Average Weekly } \\
Throughput \\
June 2012-November 2012 \\
\hline Container Types & $\begin{array}{c}\text { Average Total } \\
\text { Weekly Throughput* }\end{array}$ & $\begin{array}{c}\text { Range of Individual } \\
\text { Carrier Responses }\end{array}$ \\
\hline 20ft & 714 & $3 \ldots 150$ \\
40ft & 901 & $56 \ldots 250$ \\
40ft High Cubes & 1,539 & $87 \ldots 400$ \\
40ft Refrigerated & 113 & $0 \ldots 36$ \\
20ft Refrigerated & 17 & $0 . . .10$ \\
\hline
\end{tabular}

*Data reflect the sum of the individual carriers' average weekly throughput over a 6month timeframe 


\section{Norfolk, VA}

Norfolk

20ft Dry

\begin{tabular}{|c|c|c|c|c|c|c|c|c|c|c|}
\hline \multirow[b]{2}{*}{ Current Week: Oct 9-15 } & \multicolumn{9}{|c|}{ Carrier Availability Category Responses } & \multirow{2}{*}{$\begin{array}{l}\text { Total } \\
159\end{array}$} \\
\hline & 133 & 65 & 10 & 8 & -10 & -47 & . & . & . & \\
\hline Week 2: Oct 16-22 & 214 & 92 & 10 & 9 & 7 & -147 & . & . & . & 185 \\
\hline Week 3: Oct 23-29 & 329 & 133 & 10 & 11 & 4 & -131 & . & . & . & 356 \\
\hline \multicolumn{11}{|c|}{ 40ft Dry } \\
\hline & \multicolumn{9}{|c|}{ Carrier Availability Category Responses } & Total \\
\hline Current Week: Oct 9-15 & 212 & 80 & 12 & 9 & -2 & -10 & . & . & . & 301 \\
\hline Week 2: Oct 16-22 & 261 & 80 & 10 & 18 & 20 & 38 & . & . & . & 427 \\
\hline Week 3: Oct 23-29 & 321 & 80 & 10 & 62 & 15 & 86 & . & . & . & 574 \\
\hline \multicolumn{11}{|c|}{ 40ft High Cube } \\
\hline & \multicolumn{9}{|c|}{ Carrier Availability Category Responses } & Total \\
\hline Current Week: Oct 9-15 & 400 & 351 & 209 & 65 & 12 & -146 & . & . & . & 891 \\
\hline Week 2: Oct 16-22 & 400 & 538 & 303 & 60 & 9 & -62 & . & . & . & 1,248 \\
\hline Week 3: Oct 23-29 & 400 & 589 & 420 & 39 & 9 & -53 & . & . & . & 1,404 \\
\hline \multicolumn{11}{|c|}{ 40ft Refrigerated } \\
\hline & \multicolumn{9}{|c|}{ Carrier Availability Category Responses } & Total \\
\hline Current Week: Oct 9-15 & 270 & 96 & 47 & 10 & 4 & -16 & . & . & . & 411 \\
\hline Week 2: Oct 16-22 & 225 & 104 & 46 & 10 & 3 & -13 & . & . & . & 375 \\
\hline Week 3: Oct 23-29 & 186 & 114 & 55 & 10 & 1 & -22 & . & . & . & 344 \\
\hline \multicolumn{11}{|c|}{ 20ft Refrigerated } \\
\hline & \multicolumn{9}{|c|}{ Carrier Availability Category Responses } & Total \\
\hline Current Week: Oct 9-15 & 19 & 2 & 1 & & . & . & . & . & . & 22 \\
\hline Week 2: Oct 16-22 & 19 & 1 & 1 & . & . & . & . & . & . & 21 \\
\hline Week 3: Oct 23-29 & 19 & 2 & 1 & & & . & . & . & . & 22 \\
\hline
\end{tabular}

Note: "." denotes no response from carriers.

\section{Six-Month Snapshot of Average Weekly}

Throughput

June 2012-November 2012

\begin{tabular}{|l|c|c|}
\hline Container Types & $\begin{array}{c}\text { Average Total } \\
\text { Weekly Throughput* }\end{array}$ & $\begin{array}{c}\text { Range of Individual } \\
\text { Carrier Responses }\end{array}$ \\
\hline 20ft & 338 & $1 . .175$ \\
40ft & 797 & $8 \ldots 200$ \\
40ft High Cubes & 1,035 & $74 \ldots 300$ \\
40ft Refrigerated & 139 & $0 \ldots 58$ \\
20ft Refrigerated & 6 & $0 . . .5$ \\
\hline
\end{tabular}

*Data reflect the sum of the individual carriers' average weekly throughput over a 6month timeframe 


\section{Oakland, CA}

\begin{tabular}{|c|c|c|c|c|c|c|c|c|c|c|}
\hline \multicolumn{11}{|c|}{ Oakland } \\
\hline \multicolumn{11}{|c|}{$20 \mathrm{ft}$ Dry } \\
\hline & \multicolumn{9}{|c|}{ Carrier Availability Category Responses } & Total \\
\hline Current Week: Oct 9-15 & 243 & 100 & 56 & 26 & 12 & -29 & . & . & . & 408 \\
\hline Week 2: Oct 16-22 & 215 & 100 & 65 & -24 & 12 & -42 & . & . & . & 326 \\
\hline Week 3: Oct 23-29 & 201 & 100 & 75 & -4 & 14 & -46 & . & . & . & 340 \\
\hline \multicolumn{11}{|c|}{$40 f$ Dry } \\
\hline & \multicolumn{9}{|c|}{ Carrier Availability Category Responses } & Total \\
\hline Current Week: Oct 9-15 & 467 & 99 & 90 & 67 & 22 & 10 & . & . & . & 755 \\
\hline Week 2: Oct 16-22 & 178 & 111 & 90 & 59 & 30 & 9 & . & . & . & 477 \\
\hline Week 3: Oct 23-29 & 15 & 123 & 90 & 51 & 22 & 14 & . & . & . & 315 \\
\hline \multicolumn{11}{|c|}{ 40ft High Cube } \\
\hline & \multicolumn{9}{|c|}{ Carrier Availability Category Responses } & Total \\
\hline Current Week: Oct 9-15 & 600 & 543 & 158 & 130 & 10 & -159 & . & . & . & 1,282 \\
\hline Week 2: Oct 16-22 & 600 & 515 & 90 & 203 & 15 & -122 & . & . & . & 1,301 \\
\hline Week 3: Oct 23-29 & 600 & 432 & 41 & 253 & 20 & -13 & . & . & . & 1,333 \\
\hline \multicolumn{11}{|c|}{ 40ft Refrigerated } \\
\hline & \multicolumn{9}{|c|}{ Carrier Availability Category Responses } & Total \\
\hline Current Week: Oct 9-15 & 113 & 107 & 30 & 1 & -33 & -130 & . & . & . & 88 \\
\hline Week 2: Oct 16-22 & 66 & 107 & 30 & 1 & -73 & -135 & . & . & . & -4 \\
\hline Week 3: Oct 23-29 & 14 & 108 & 30 & 1 & -63 & -144 & . & . & . & -54 \\
\hline \multicolumn{11}{|c|}{ 20ft Refrigerated } \\
\hline & \multicolumn{9}{|c|}{ Carrier Availability Category Responses } & Total \\
\hline Current Week: Oct 9-15 & 10 & 8 & 7 & & . & . & . & . & . & 25 \\
\hline Week 2: Oct 16-22 & 10 & 10 & 7 & . & . & . & . & . & . & 27 \\
\hline Week 3: Oct 23-29 & 10 & 12 & 65 & & & . & . & . & . & 87 \\
\hline
\end{tabular}

Note: "." denotes no response from carriers.

\begin{tabular}{|l|c|c|}
\hline \multicolumn{3}{|c|}{ Six-Month Snapshot of Average Weekly } \\
Throughput \\
June 2012-November 2012 \\
\hline Container Types & Average Total & Range of Individual \\
& Weekly Throughput* & Carrier Responses \\
\hline 20ft & 853 & $26 \ldots .250$ \\
40ft & 899 & $14 . .300$ \\
40ft High Cubes & 1,199 & $34 . . .400$ \\
40ft Refrigerated & 358 & $26 \ldots 120$ \\
20ft Refrigerated & 24 & $0 . . .8$ \\
\hline
\end{tabular}

*Data reflect the sum of the individual carriers' average weekly throughput over a 6month timeframe 


\section{Savannah, GA}

\begin{tabular}{|c|c|c|c|c|c|c|c|c|c|c|}
\hline \multicolumn{11}{|c|}{ Savannah } \\
\hline \multicolumn{11}{|c|}{ 20ft Dry } \\
\hline & \multicolumn{9}{|c|}{ Carrier Availability Category Responses } & Total \\
\hline Current Week: Oct 9-15 & 180 & 104 & 91 & 4 & 2 & -11 & & . & . & 370 \\
\hline Week 2: Oct 16-22 & 180 & 207 & 103 & 45 & 3 & -25 & & . & . & 513 \\
\hline Week 3: Oct 23-29 & 180 & 309 & 76 & 38 & 3 & -71 & & . & . & 535 \\
\hline \multicolumn{11}{|c|}{ 40ft Dry } \\
\hline & \multicolumn{9}{|c|}{ Carrier Availability Category Responses } & Total \\
\hline Current Week: Oct 9-15 & 211 & 177 & 150 & 6 & -11 & -433 & & . & . & 100 \\
\hline Week 2: Oct 16-22 & 286 & -40 & 150 & 7 & -55 & -553 & & . & . & -205 \\
\hline Week 3: Oct 23-29 & 402 & -45 & 150 & 4 & -60 & -668 & & . & . & -217 \\
\hline \multicolumn{11}{|c|}{ 40ft High Cube } \\
\hline & \multicolumn{9}{|c|}{ Carrier Availability Category Responses } & Total \\
\hline Current Week: Oct 9-15 & 469 & 371 & 350 & 0 & -89 & -511 & & . & . & 590 \\
\hline Week 2: Oct 16-22 & 359 & 579 & 350 & 0 & -59 & -598 & & . & . & 631 \\
\hline Week 3: Oct 23-29 & 367 & 788 & 350 & 0 & -55 & -694 & & . & . & 756 \\
\hline \multicolumn{11}{|c|}{ 40ft Refrigerated } \\
\hline & \multicolumn{9}{|c|}{ Carrier Availability Category Responses } & Total \\
\hline Current Week: Oct 9-15 & 300 & 158 & 135 & 13 & 10 & -20 & & . & . & 596 \\
\hline Week 2: Oct 16-22 & 325 & 154 & 110 & 54 & 10 & -80 & . & . & . & 573 \\
\hline Week 3: Oct 23-29 & 350 & 154 & 117 & 91 & 10 & -65 & & . & . & 657 \\
\hline \multicolumn{11}{|c|}{ 20ft Refrigerated } \\
\hline & \multicolumn{9}{|c|}{ Carrier Availability Category Responses } & Total \\
\hline Current Week: Oct 9-15 & 4 & 1 & -1 & . & . & . & & . & . & 4 \\
\hline Week 2: Oct 16-22 & 3 & 1 & -2 & . & . & . & . & . & . & 2 \\
\hline Week 3: Oct 23-29 & 2 & 1 & -3 & . & . & . & . & . & . & 0 \\
\hline
\end{tabular}

Note: "." denotes no response from carriers.

\begin{tabular}{|l|c|c|}
\hline \multicolumn{3}{|c|}{ Six-Month Snapshot of Average Weekly } \\
Throughput \\
June 2012-November 2012 \\
\hline \multirow{2}{*}{ Container Types } & $\begin{array}{c}\text { Average Total } \\
\text { Weekly Throughput* }\end{array}$ & $\begin{array}{c}\text { Range of Individual } \\
\text { Carrier Responses }\end{array}$ \\
\hline $\mathbf{2 0 f t}$ & 449 & $0 \ldots 150$ \\
$\mathbf{4 0 f t}$ & 982 & $0 . .409$ \\
$\mathbf{4 0 f t}$ High Cubes & 1,450 & $21 \ldots 385$ \\
$\mathbf{4 0 f t}$ Refrigerated & 221 & $0 \ldots 80$ \\
$\mathbf{2 0 f t}$ Refrigerated & 4 & $0 . .2$ \\
\hline
\end{tabular}

*Data reflect the sum of the individual carriers' average weekly throughput over a 6month timeframe 


\section{Seattle, WA}

\begin{tabular}{|c|c|c|c|c|c|c|c|c|c|c|}
\hline \multicolumn{11}{|c|}{ Seattle } \\
\hline \multicolumn{11}{|c|}{ 20ft Dry } \\
\hline & \multicolumn{9}{|c|}{ Carrier Availability Category Responses } & Total \\
\hline Current Week: Oct 9-15 & 184 & 100 & 18 & 10 & . & . & . & . & . & 312 \\
\hline Week 2: Oct 16-22 & 177 & 100 & 33 & 9 & . & . & . & . & . & 319 \\
\hline Week 3: Oct 23-29 & 170 & 100 & 38 & 10 & . & . & . & . & . & 318 \\
\hline \multicolumn{11}{|c|}{$40 f t$ Dry } \\
\hline & \multicolumn{9}{|c|}{ Carrier Availability Category Responses } & Total \\
\hline Current Week: Oct 9-15 & 184 & 108 & 40 & 8 & . & . & . & . & . & 340 \\
\hline Week 2: Oct 16-22 & 163 & 96 & 40 & 9 & . & . & . & . & . & 308 \\
\hline Week 3: Oct 23-29 & 141 & 77 & 40 & 7 & . & . & . & . & . & 265 \\
\hline \multicolumn{11}{|c|}{ 40ft High Cube } \\
\hline & \multicolumn{9}{|c|}{ Carrier Availability Category Responses } & Total \\
\hline Current Week: Oct 9-15 & 175 & 60 & 8 & 7 & . & . & . & . & . & 250 \\
\hline Week 2: Oct 16-22 & 175 & 34 & 8 & -28 & . & . & . & . & . & 189 \\
\hline Week 3: Oct 23-29 & 175 & 30 & 8 & -78 & . & . & . & . & . & 135 \\
\hline \multicolumn{11}{|c|}{ 40ft Refrigerated } \\
\hline & \multicolumn{9}{|c|}{ Carrier Availability Category Responses } & Total \\
\hline Current Week: Oct 9-15 & 26 & 22 & 22 & 20 & . & . & . & . & . & 90 \\
\hline Week 2: Oct 16-22 & -24 & 15 & 20 & 20 & . & . & . & . & . & 31 \\
\hline Week 3: Oct 23-29 & -110 & 3 & 20 & 20 & . & . & . & . & . & -67 \\
\hline \multicolumn{11}{|c|}{ 20ft Refrigerated } \\
\hline & \multicolumn{9}{|c|}{ Carrier Availability Category Responses } & Total \\
\hline Current Week: Oct 9-15 & 5 & 1 & . & & . & . & . &. & . & 6 \\
\hline Week 2: Oct 16-22 & 4 & 1 & . & . & . & . & . & . & . & 5 \\
\hline Week 3: Oct 23-29 & 3 & 1 & & & . & . & . & . & . & 4 \\
\hline
\end{tabular}

Note: "." denotes no response from carriers.

\begin{tabular}{|l|c|c|}
\hline \multicolumn{3}{|c|}{ Six-Month Snapshot of Average Weekly } \\
Throughput \\
June 2012-November 2012 \\
\hline Container Types & $\begin{array}{c}\text { Average Total } \\
\text { Weekly Throughput* }\end{array}$ & $\begin{array}{c}\text { Range of Individual } \\
\text { Carrier Responses }\end{array}$ \\
\hline 20ft & 172 & $29 . .70$ \\
40ft & 263 & $7 \ldots 91$ \\
40ft High Cubes & 462 & $104 \ldots 148$ \\
40ft Refrigerated & 172 & $0 . .122$ \\
20ft Refrigerated & 7 & $0 . .5$ \\
\hline
\end{tabular}

*Data reflect the sum of the individual carriers' average weekly throughput over a 6month timeframe 


\section{Tacoma, WA}

\begin{tabular}{|c|c|c|c|c|c|c|c|c|c|c|}
\hline \multicolumn{11}{|c|}{ Tacoma } \\
\hline \multicolumn{11}{|c|}{ 20ft Dry } \\
\hline & \multicolumn{9}{|c|}{ Carrier Availability Category Responses } & Total \\
\hline Current Week: Oct 9-15 & 229 & 16 & 10 & 5 & -17 & . & . & . & . & 243 \\
\hline Week 2: Oct 16-22 & 161 & 16 & 10 & 5 & -17 & . & . & . & . & 175 \\
\hline Week 3: Oct 23-29 & 138 & 14 & 10 & 5 & -7 & . & . & . & . & 160 \\
\hline \multicolumn{11}{|c|}{ 40ft Dry } \\
\hline & \multicolumn{9}{|c|}{ Carrier Availability Category Responses } & Total \\
\hline Current Week: Oct 9-15 & 418 & 67 & 8 & -15 & -223 & . & . & . & . & 255 \\
\hline Week 2: Oct 16-22 & 288 & 70 & 8 & -15 & -73 & . & . & . & . & 278 \\
\hline Week 3: Oct 23-29 & 300 & 38 & 8 & -15 & -80 & . & . & . & . & 251 \\
\hline \multicolumn{11}{|c|}{ 40ft High Cube } \\
\hline & \multicolumn{9}{|c|}{ Carrier Availability Category Responses } & Total \\
\hline Current Week: Oct 9-15 & 303 & 40 & 8 & 7 & -494 & . & . & . & . & -136 \\
\hline Week 2: Oct 16-22 & 233 & 40 & 10 & -22 & -276 & . & . & . & . & -15 \\
\hline Week 3: Oct 23-29 & 593 & 40 & 10 & -208 & -285 & . & . & . & . & 150 \\
\hline \multicolumn{11}{|c|}{ 40ft Refrigerated } \\
\hline & \multicolumn{9}{|c|}{ Carrier Availability Category Responses } & Total \\
\hline Current Week: Oct 9-15 & 213 & 106 & 5 & 0 & -245 & . & . & . & . & 79 \\
\hline Week 2: Oct 16-22 & 126 & -107 & 5 & 0 & -247 & . & . & . & . & -223 \\
\hline Week 3: Oct 23-29 & 156 & -367 & 5 & 0 & -249 & . & . & . & . & -455 \\
\hline \multicolumn{11}{|c|}{ 20ft Refrigerated } \\
\hline & \multicolumn{9}{|c|}{ Carrier Availability Category Responses } & Total \\
\hline Current Week: Oct 9-15 & 11 & 1 & . & . & . & . & . & . & . & 12 \\
\hline Week 2: Oct 16-22 & 13 & 1 & . & . & . & . & . & . & . & 14 \\
\hline Week 3: Oct 23-29 & 14 & 1 & . & . & & . & . & . & . & 15 \\
\hline
\end{tabular}

Note: "." denotes no response from carriers.

\begin{tabular}{|l|c|c|}
\hline \multicolumn{3}{|c|}{ Six-Month Snapshot of Average Weekly } \\
Throughput \\
June 2012-November 2012 \\
\hline Container Types & Average Total & Range of Individual \\
& Weekly Throughput* & Carrier Responses \\
\hline 20ft & 383 & $1 \ldots 300$ \\
40ft & 671 & $15 . . .500$ \\
40ft High Cubes & 797 & $76 \ldots 600$ \\
40ft Refrigerated & 493 & $0 . .203$ \\
20ft Refrigerated & 4 & $0 . .4$ \\
\hline
\end{tabular}

*Data reflect the sum of the individual carriers' average weekly throughput over a 6month timeframe 


\section{Understanding this Report}

The weekly Ocean Shipping Container Availability Report (OCSAR) provides a snapshot of container availability for westbound transpacific trade lanes at select intermodal locations around the country. The data presented in this report are gathered from 6 member carriers in the Transpacific Stabilization Agreement (TSA). The carriers include COSCO, Evergreen, Hanjin Shipping, Hapag Lloyd, Yang Ming Transport Corporation, and OOCL.

- The data are estimates of container availability per week by the participating carriers. The carriers determine container availability by comparing current supplies at each of the 18 intermodal locations with the demand for these containers based on up-to-date bookings or reservation information in the westbound transpacific trade lane.

- $\quad$ Each week, participating carriers report the estimated number of containers available for the current week (W1) as well as estimates for the subsequent 2 weeks (W2 and W3).

- Maps on pages 3-5 offer a national overview of weekly container availability, as well as an average of availability over the past 6 months. The weekly national data are derived from the sum of the individual carrier responses at each intermodal location.

- Individual carrier responses are presented by location on pages 6-23 and have been sorted from largest to smallest. To maintain carrier confidentiality, specific carrier identification is not provided.

- Carrier responses showing negative numbers, such as -29 , should be interpreted as meaning that 29 containers are needed by shippers but the carrier is not able to supply them. A positive number, such as 64 , would mean 64 containers are available that are not demanded. An indication of 0 means the carriers have the exact amount of containers needed based on current supply and demand estimates.

- In addition to the weekly container availability data, the participating carriers provide average weekly throughput data for the past 6 months. These data help readers understand the aggregate flow of containers that have moved through the 18 select locations by the 8 participating carriers over the previous 6 months. These data will only be updated every 6 months.

- Availability levels are subject to change, and may be affected by delays in return of equipment to carriers by shippers or consignees, and by free time restrictions imposed by railroads. Due to the dynamic nature of the container industry, carriers cannot be held responsible for the level of availability at the time of inquiry.

- $\quad$ Data presented in the report do not imply availability of chassis.

- Container availability estimates represent availability for all export cargo, not just agricultural products.

- USDA collects these data on a voluntary basis and cannot be held responsible for the status of container availability when a reader makes inquiry with an ocean carrier. USDA relies on the participating ocean carriers to ensure the data is accurate.

- Terminology:

20ft Dry Container (20ft): A standard shipping container that is 20 feet long.

40ft Dry Container (40ft): A standard shipping container that is 40 feet long.

40ft High-Cube (HC): A shipping container which is 40 feet long and 9 feet, 6 inches tall (1 foot taller than a standard $40 \mathrm{ft}$ dry container).

40ft reefer: A refrigerated shipping container which is 40 feet long.

20ft reefer: A refrigerated shipping container which is 20 feet long.

Chassis: A wheeled frame to which a shipping container can be locked for storage or movement. (Plural chassis) 


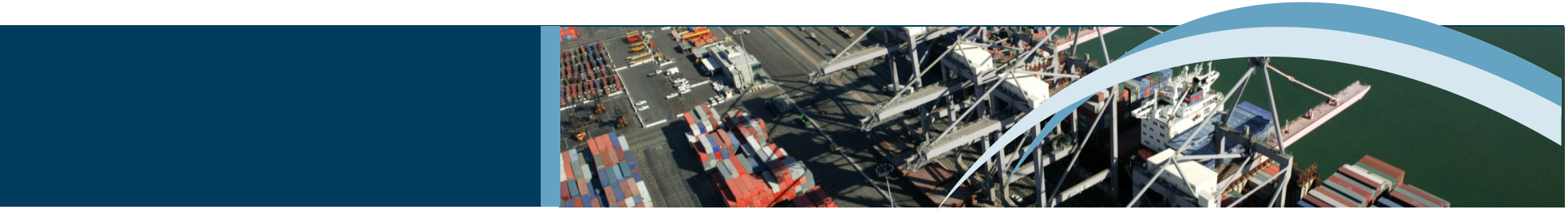

The U.S. Department of Agriculture (USDA) prohibits discrimination in all of its programs and activities on the basis of race, color, national origin, age, disability, and where applicable, sex (including gender identity and expression), marital status, familial status, parental status, religion, sexual orientation, political beliefs, genetic information, reprisal, or because all or part of an individual's income is derived from any public assistance program. (Not all prohibited bases apply to all programs.) Persons with disabilities who require alternative means for communication of program information (Braille, large print, audiotape, etc.) should contact USDA's TARGET Center at (202) 720-2600 (voice and TDD).

To file a complaint of discrimination, write to USDA, Director, Office of Civil Rights, 1400 Independence Avenue, S.W., Washington, D.C. 20250-9410, or call (800) 795-3272 (voice) or (202) 720-6382 (TDD). USDA is an equal opportunity provider and employer.

\section{Preferred citation:}

U.S. Dept. of Agriculture, Agricultural Marketing Service. Ocean Shipping Container Availability Report. October 9, 2013. Web. <http://dx.doi.org/10.9752/TS057.10-9-2013> 